\title{
Discussões sobre o \\ dimensionamento de vigas de concreto armadas à \\ flexão com barras de GFRP
}

\begin{tabular}{c}
\hline GLÁUCIA MARIA DALFRÉ - Professora \\
\hline AMANDA DUARTE ESCOBAL MAZZÚ - ENGENHEIRA CIVIL, Doutoranda \\
\hline FERNANDA GIANNOTTI DA SILVA FERREIRA - ProfesSora \\
\hline Unversidade Federal de São Carlos (PPGECII/UFSCAR)
\end{tabular}

\section{RESUMO}

O TRABALHO APRESENTA O DIMENSIONAMENTO À FLEXÃO DE VIGAS BIAPOIADAS DE CONCRETO ARMADAS OU COM BARRAS DE AÇO CONVENCIONAIS OU COM POLÍMEROS REFORÇADOS COM FIBRAS DE VIDRO (GFRP), CUJOS RESULTADOS FORAM COMPARADOS AOS OBTIDOS EM ENSAIOS. OS AUTORES CONCLUÍRAM QUE: O DIMENSIONAMENTO BASEADO NAS NORMAS ABNT NBR 6118 (2014) E ACl 318 (2019) É EFICAZ PARA DETERMINAR A CAPACIDADE DE

CARGA DAS VIGAS E SEU MODO DE RUPTURA; E OS MODELOS APRESENTADOS PERMITEM O DIMENSIONAMENTO SEGURO DOS ELEMENTOS DE CONCRETO ARMADOS COM BARRAS DE GFRP. ESTE TRABALHO É A SEGUNDA PARTE DO ARTIGO "DIMENSIONAMENTO DE VIGAS DE CONCRETO ARMADAS À FLEXÃO COM BARRAS NÃO METÁLICAS", PUBLICADO NA EDIÇÃO 98 DA CONCRETO \& CONSTRUÇÕES.

Palavras-chave: dimensionamento à flexão de vigas; concreto armado; polímeros reforçados com fibras de vidro; segurança das estruturas.

\section{INTRODUÇÃO}

$\mid \begin{aligned} & \text { o Brasil, é comum a utiliza- } \\ & \text { ção de estruturas de con- } \\ & \text { creto armado com barras de }\end{aligned}$ aço, fato que se deve, segundo Lima e Prado (2014), ao seu menor custo de execução quando comparado aos custos de execução de estruturas metálicas ou pré-moldadas, além da existência de normas bem definidas para seu projeto e execução. Entretanto, tais estruturas comumente apresentam problemas relacionados à corrosão das armaduras metálicas pelo ataque de cloretos ou pela carbonatação, gerando a necessidade do estudo de novas tecnologias e materiais. Neste contexto, inserem-se os polímeros reforçados com fibras (de sigla internacional FRP - Fiber Reinforced Polymer), materiais de elevada resistência mecânica, excelente resistência à corrosão e magneticamente neutros (ISSA et al., 2011), capazes de substituir os materiais convencionais utilizados nas técnicas de reforço estrutural e o aço utilizado como armadura em estruturas de concreto armado.

Os polímeros reforçados ou estruturados com fibras de carbono (CFRP - Carbon Fiber Reinforced Polymer), de vidro (GFRP - Glass Fiber Reinforced Polymer), aramida (AFRP - Aramid Fiber Reinforced Polymer) e basalto (BFRP - Basalt Fiber Reinforced Polymer) são os mais utilizados.
Caso sejam corretamente projetados e fabricados, a utilização do FRP pode prover uma vida útil mais longa e menores custos de manutenção do que quando se utilizam armaduras de aço convencional em estruturas de concreto armado (ACl 440.9R, 2015).

O objetivo principal deste trabalho é obter um melhor entendimento do comportamento à flexão de vigas armadas com barras de aço convencional e barras de GFRP. Assim, espera-se contribuir com o avanço do conhecimento acerca da utilização de barras de FRP como armadura em estruturas de concreto armado, principalmente para a confecção de elementos em ambientes de elevada agressividade ambiental. 
Para informações sobre o estado da arte, consultar artigo na edição 98 da CONCRETO \& Construções.

\section{MATERIAIS E MÉTODOS}

Um estudo analítico do comportamento de duas vigas biapoiadas de concreto armado com barras de aço e de duas vigas biapoiadas de concreto armado com barras de GFRP foi realizado. Posteriormente, os resultados analíticos foram comparados aos ensaios recentemente realizados por Mazzú (2020). Note-se que o trabalho aqui apresentado ainda encontra-se em andamento e também avaliará o comportamento de tais elementos quando expostos ao ingresso de cloretos. A Figura 1 apresenta o esquema do ensaio de flexão em três pontos e os diagramas de momento fletor e esforço cortante, enquanto as características geométricas e as armaduras longitudinais das vigas encontram-se representadas na Figura 2.

As quatro vigas foram confecciona-

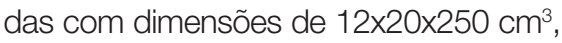
vão livre entre os apoios de $230 \mathrm{~cm}$ e concreto com resistência característica à compressão de 32,8 MPa, valor experimentalmente obtido. Duas vigas foram produzidas com armaduras longitudinais positivas compostas por 2 barras de aço CA-50, com diâmetro de 10 mm (taxa de armadura longitudinal de 0,75\%) e duas com armaduras longitudinais positivas compostas por 2 barras de fibra de vidro (GFRP) com diâmetro de 10 mm, tensão de ruptura $\left(f_{f u}^{*}\right)$, segundo o fabricante, de $1047 \mathrm{MPa}$, módulo de elasticidade $\left(E_{f}\right)$ de $48 \mathrm{GPa}$ e deformação na ruptura estimada em 21,8\% (Figura 3). Todas as vigas apresentaram cobrimento de 15 $\mathrm{mm}$ e porta estribos compostos por duas barras de aço CA-50 com diâmetro de 6,3 mm. Como este estudo contempla a

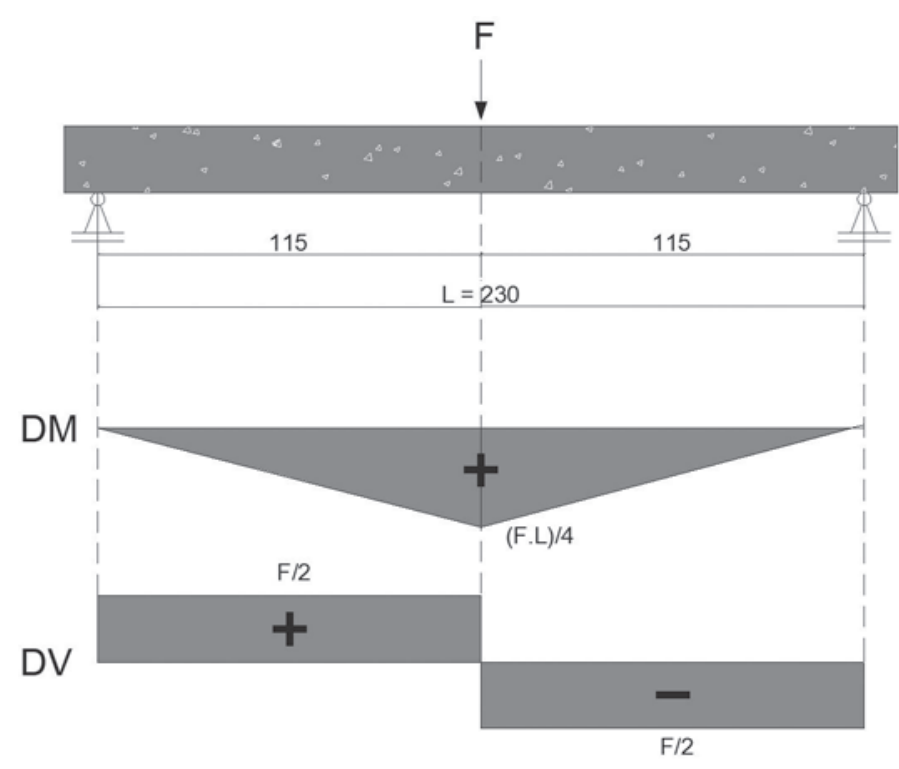

Figura 1

Carregamento e diagramas de momento fletor (DM) e esforço cortante (DV). Medidas em centímetros

Fonte: 0 autor

avaliação de barras de GFRP à flexão e para se evitar a ruptura por cisalhamento, estribos de aço CA-60, com diâmetro de $5 \mathrm{~mm}$, foram espaçados a cada $10 \mathrm{~cm}$ em todas as vigas, conforme ilustrado na Figura 2.

Para as vigas armadas com barras de GFRP, o dimensionamento foi realizado utilizando-se o processo de dimensionamento proposto pelo $\mathrm{ACl}$ 440.1R (2015), considerando-se vigas não expostas à água e intempéries, sendo, portanto, $\mathrm{C}_{\mathrm{E}}$ (fator ambiental de redução) igual a 0,8.

Com relação à viga armada com barras de aço, a capacidade resistente foi determinada com base nas disposições da ABNT NBR 6118 (2014) e do ACl 318 (2019). Para o dimensionamento das vigas armadas com barras de aço, a ABNT NBR 6118 (2014) utiliza coeficientes de minoração das propriedades dos materiais, nomeadamente, resistência à compressão do concreto e resistência ao escoamento do aço, enquanto a ACl 318 (2019) não utiliza coeficiente de minoração das propriedades, entretanto, reduz a capacidade resistente obtida por um coeficiente de segurança determinado com base no tipo de estribo utilizado e na deformação apresentada pelo aço. Para o dimensionamento das vigas armadas com barras de GFRP, o ACl 440.1R (2015) apresenta procedimentos baseados no ACI 318 (2019), ou seja, a capacidade resistente obtida é reduzida por um coeficiente de segurança determinado com base no tipo de ruína obtida. Assim, neste trabalho, os cálculos de capacidade resistente das vigas armadas com barras de GFRP foram realizados com e sem a utilização dos coeficientes de redução apresentados pelos códigos de dimensionamento utilizados de forma a se obter valores de projetos e, também, simular os resultados obtidos em laboratório.

\section{MODELOS DE DIMENSIONAMENTO E RESULTADOS}

\section{I Dimensionamento}

Apresentam-se aqui os modelos 
de dimensionamento utilizados para o cálculo das vigas de concreto armado

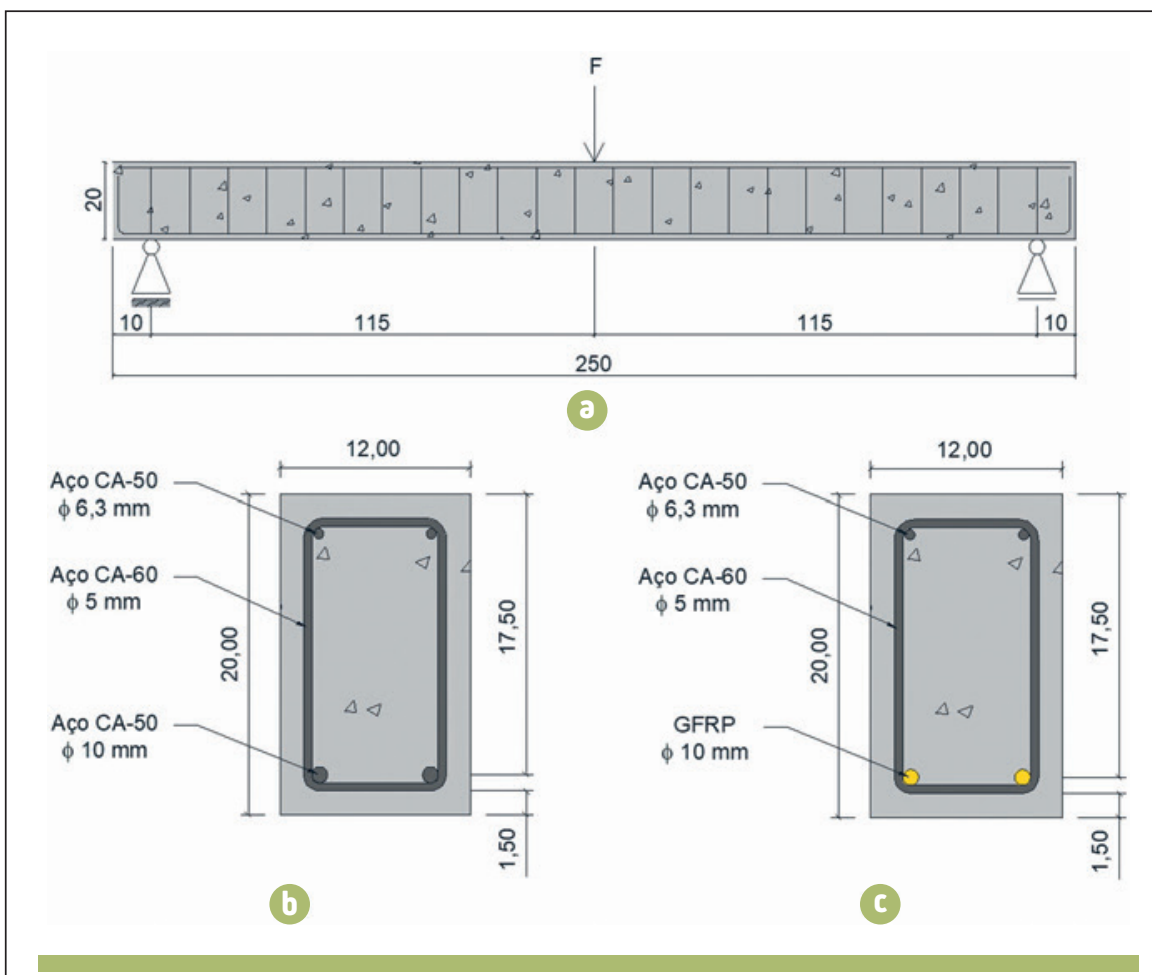

Figura 2

(a) Viga de concreto armado utilizada no programa experimental, seção transversal com (b) aço convencional e (c) barras de GFRP. Dimensões em centímetros

Fonte: Mazzú, 2020
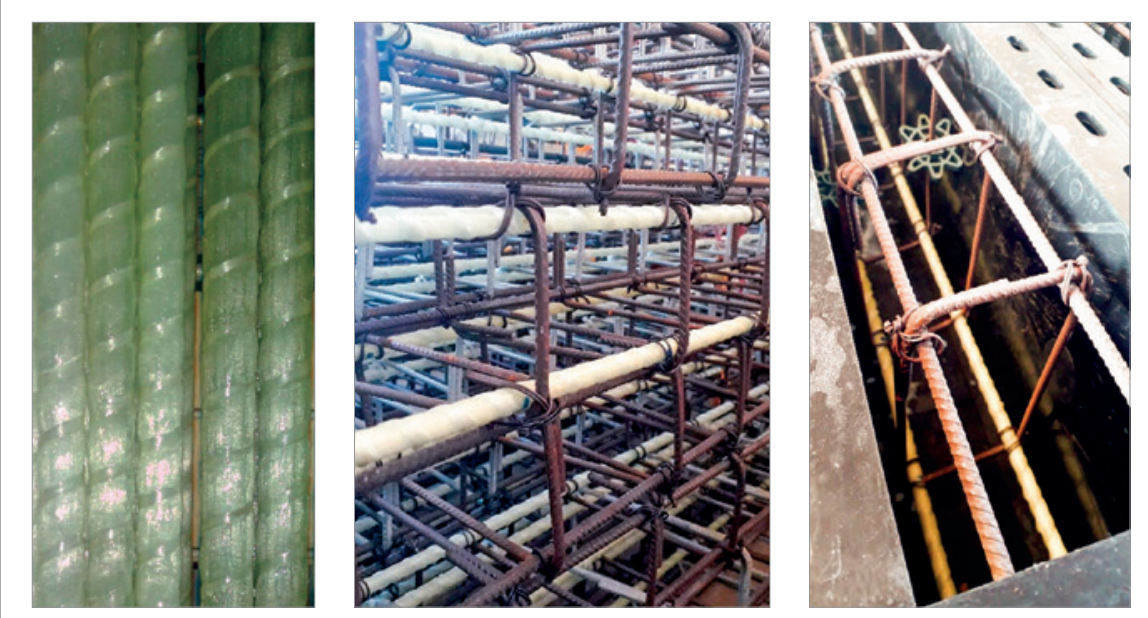

Figura 3

Armadura de GFRP utilizada

Fonte: Mazzú, 2020
NBR 6118 (2014) e ACl 318 (2019), assim como das vigas armadas com barras de GFRP, as quais foram dimensionadas segundo a $\mathrm{ACl} 440.1 \mathrm{R}$ (2015).

\section{I.I ABNT NBR 6II8 (2014)}

A determinação da resistência à flexão das vigas de concreto armadas com barras de aço foi baseada na ABNT NBR 6118 (2014), com o uso do diagrama simplificado de distribuição de tensões e deformações de uma viga de seção retangular com armadura simples (Figura 4).

A determinação da profundidade de linha neutra é obtida com o uso da Equação 1, onde $A_{s}$ é a área da armadura longitudinal $(2 \phi 10 \mathrm{~mm}$, perfazendo uma área de 1,57 cm²), f é a tensão de escoamento do aço (500 MPa), d é a altura útil $(17,5 \mathrm{~cm}), \lambda$ é o valor definido em 0,8 para a altura do diagrama retangular simplificado da distribuição de tensões do concreto comprimido com resistência à compressão de até 50 $\mathrm{MPa}, \mathrm{x}$ é a profundidade da linha neutra, $b_{w}$ é a largura da seção transversal, $\alpha_{c}$ é igual a 0,85 da tensão máxima de compressão para concretos até

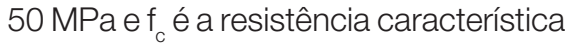
do concreto (32,8 MPa). Em situações de projeto, no cálculo da profundidade da linha neutra, a resistência característica do concreto e a tensão de escoamento do aço são reduzidas por coeficientes de minoração iguais a 1,4 e 1,15 , respectivamente.

$x=\frac{A_{s} \cdot f_{y}}{\alpha_{c} \cdot \lambda \cdot b_{w} \cdot f_{c}}$
$x=\frac{1,57 \mathrm{~cm}^{2} \cdot\left(\frac{50 \mathrm{kN} / \mathrm{cm}^{2}}{1,15}\right)}{0,85 \cdot 0,8 \cdot 0,12 \cdot\left(\frac{32800 \mathrm{kN} / \mathrm{m}^{2}}{1,4}\right)}=0,0357 \mathrm{~m}$

Considerando-se os conceitos de domínio da ABNT NBR 6118 (2014) 
e sabendo-se que no limite entre os domínios 2 e 3 o aço assume uma deformação igual a 10 \% e o concreto assume seu valor de deformação última (3,5 \%o), por meio de semelhança de triângulos é possível encontrar o valor de profundidade de linha neutra limite entre os domínios 2 e 3 (Equação 2).

$\frac{x_{2 / 3}}{3,5 \% 0}=\frac{d-x_{2 / 3}}{10 \% 0} \rightarrow x_{2 / 3}=0,259 \cdot d$
$x_{2 / 3}=0,259 \cdot d=0,259 \cdot 17,5=4,53 \mathrm{~cm}=0,0453 \mathrm{~m}$

A ABNT NBR 6118 (2014) ainda define um valor máximo para a profundidade de linha neutra para que a ductilidade seja garantida. Para vigas com concreto com resistência característica até $50 \mathrm{MPa}$, este valor é igual a $45 \%$ da altura útil, com apresentado na Equação 3.

$$
\text { [3] }
$$

$x_{\lim }=0,45 \cdot d$

$x_{\lim }=0,45 \cdot d=0,45 \cdot 17,5=7,88 \mathrm{~cm}=0,788 \mathrm{~m}$

Pela comparação do valor da profundidade de linha neutra calculada $(x)$ com a profundidade de linha neutra limite entre os domínios 2 e $3\left(x_{2 / 3}\right)$ e a profundidade de linha limite para garantia da ductilidade $\left(x_{\text {lim }}\right)$, verifica-se que 0 dimensionamento encontra-se em domínio 2 e que a ductilidade está garantida. Sabendo-se que a viga trabalha no domínio 2, tem-se a deformação do aço $\left(\varepsilon_{s}\right)$ igual a $10 \%$ e a deformação no concreto pode ser calculada por semeIhança de triângulos, de acordo com a Equação 4.

$\frac{\varepsilon_{S}}{d-x}=\frac{\varepsilon_{c}}{x} \rightarrow \frac{10}{0,175-0,0357}=\frac{\varepsilon_{c}}{0,0357} \rightarrow \varepsilon_{c}=2,6 \% 0$

Para o cálculo do máximo momento resistente, foram utilizadas as formulações apresentadas nas Equações 5 e 6, nas quais $M_{d c}$ e $M_{d t}$ são os momentos resistentes obtidos pela análise

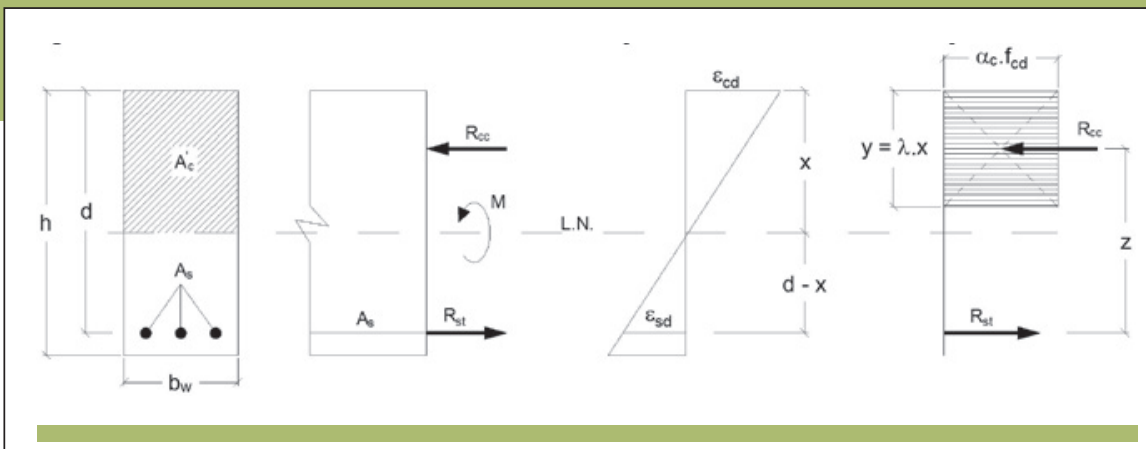

Figura 4

Diagrama de tensão e deformação de uma viga de seção retangular

Fonte: 0 Autor

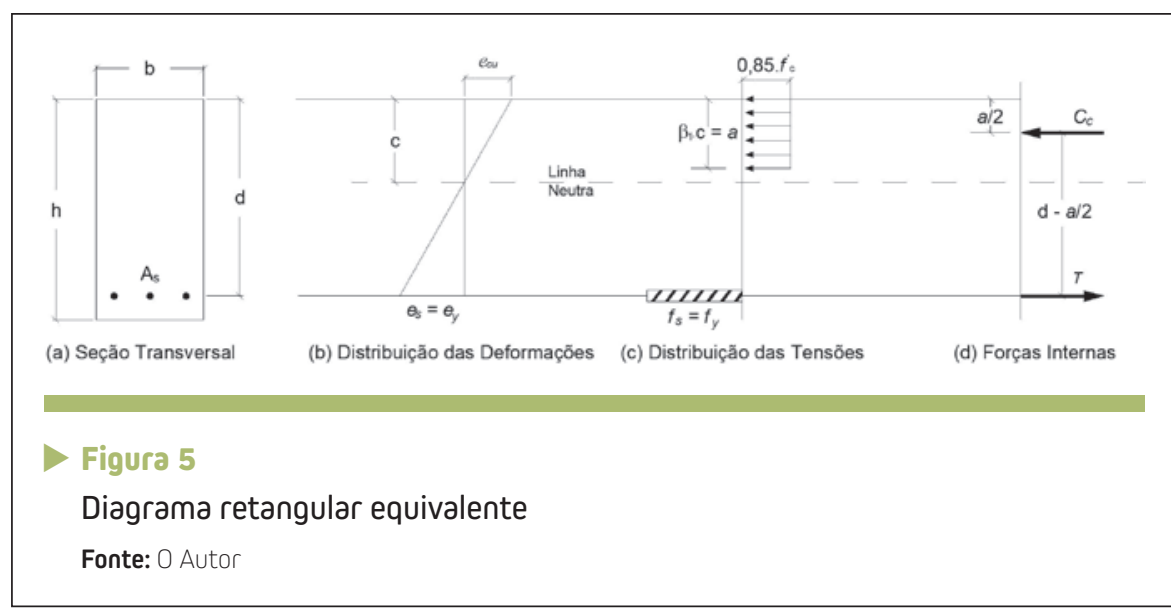

do bloco comprimido ou tracionado, respectivamente.

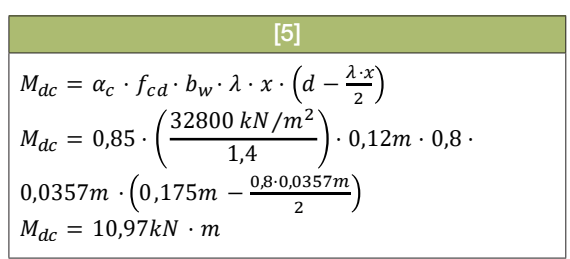

\begin{tabular}{|c|}
\hline$[6]$ \\
$M_{d t}=1,57 \mathrm{~cm}^{2} \cdot\left(\frac{50 \mathrm{kN} / \mathrm{cm}^{2}}{1,15}\right)$ \\
$\left(0,175 \mathrm{~m}-\frac{0,8 \cdot 0,0357 \mathrm{~m}}{2}\right)=10,97 \mathrm{kN} \cdot \mathrm{m}$
\end{tabular}

Tendo em vista a configuração de carregamento utilizada, este momento corresponde a uma carga concentrada, aplicada a meio vão, de 19,1 kN.

\section{I.2 ACI 318 (2019)}

Com relação ao dimensionamento segundo o $\mathrm{ACl} 318$ (2019), esta recomendação também permite como simplificação o uso de um diagrama retangular equivalente, tal como apre- senta a Figura 5. Entretanto, o dimensionamento é realizado com o uso de valores característicos de propriedades dos materiais, e a capacidade resistente é posteriormente minorada pelo fator de redução $\phi$.

Os valores de $\beta_{1}$ variam de acordo com a classe de resistência à compressão do concreto ( $\left.f_{c}^{\prime}\right)$ e possuem os valores apresentados na Equação 7. Tendo em vista a resistência à compressão de $32,8 \mathrm{MPa}$, um valor de $\beta_{1}$ igual a 0,816 foi obtido.

$\beta_{1}=\left\{\begin{array}{ccc|}0,85 & \text { para } & f_{c}^{\prime}<28 \mathrm{MPa} \\ 0,85-\frac{0,05 \cdot\left(f_{c}^{\prime}-28\right)}{7} & \text { para } & 28<f_{c}^{\prime}<55 \mathrm{MPa} \\ 0,65 & \text { para } & f_{c}^{\prime}>55 \mathrm{MPa} \\ \hline\end{array}\right.$

Tal como considerado pela ABNT NBR 6118 (2014), a profundidade do bloco retangular equivalente (a) é obtida com o uso da Equação 8, onde $A_{s}$ é a área da armadura longitudinal $\left(1,57 \mathrm{~cm}^{2}\right), \mathrm{f}_{\mathrm{y}}$ é a tensão de escoamento do aço (500 MPa), b é a largura da se- 
ção transversal e f' ${ }_{c}$ é a resistência característica do concreto (32,8 MPa).

$a=\frac{A_{s} \cdot f_{y}}{0,85 \cdot f_{c}^{\prime} \cdot b}=\frac{1,57 \mathrm{~cm}^{2} \cdot 50 \mathrm{kN} / \mathrm{cm}^{2}}{0,85 \cdot 32800 \mathrm{kN} / \mathrm{m}^{2} \cdot 0,12 \mathrm{~m}}=0,0235 \mathrm{~m}$

A profundidade da linha neutra (c) é calculada por meio da Equação 9.

$c=\frac{a}{\beta_{1}}=\frac{0,0235}{0,816}=0,0288 \mathrm{~m}$

A deformação do aço $\left(\varepsilon_{s}\right)$ é obtida a partir da compatibilidade de deformações apresentada na Equação 10. A norma ACl 318 (2019) considera que a deformação última no concreto $\left(\varepsilon_{\text {cu }}\right)$ ocorre com valor de 3\%. Assim, temos a Equação 10.

$\left[\begin{array}{c|}{[10]} \\ \varepsilon_{S}=\left(\frac{d-c}{c}\right) \cdot \varepsilon_{c u}=\left(\frac{0,175 m-0,0288 m}{0,0288}\right) \cdot 3 \% 0=15,2 \% 0\end{array}\right.$

A deformação do aço que leva ao início de seu escoamento $\left(\varepsilon_{\text {sy }}\right)$ pode ser calculada com o uso da Equação 11, considerando que o módulo de elasticidade do aço (E), pela norma americana, possui valor de $200 \mathrm{GPa}$. Desta forma, quando o aço está escoando (ou seja, $\varepsilon_{\mathrm{s}}>\varepsilon_{\mathrm{sy}}$ ), obtém-se o momento nominal resistido pela análise do bloco tracionado $\left(M_{n}\right)$, o qual é calculado por intermédio da Equação 12.

$\varepsilon_{S y}=\frac{f_{y}}{E}=\frac{500 \mathrm{MPa}}{200000 \mathrm{MPa}}=0,0025=2,5 \% 0$

\begin{tabular}{|l|}
\hline \multicolumn{1}{|c|}{} \\
\hline$M_{n}=A_{s} \cdot f_{y} \cdot\left(d-\frac{a}{2}\right)$ \\
$M_{n}=1,57 \mathrm{~cm}^{2} \cdot 50 \mathrm{kN} / \mathrm{cm}^{2}$ \\
$\left(0,175 \mathrm{~m}-\frac{0,0235 \mathrm{~m}}{2}\right)=12,82 \mathrm{kN} \cdot \mathrm{m}$ \\
\hline
\end{tabular}

O momento resistente de cálculo $\left(M_{u}\right)$ é obtido pelo produto entre o momento nominal e o fator de redução $\phi$, tal como apresentado nas Equações 13 e 14. Para a determinação de $\phi, \varepsilon_{\mathrm{s}}$ é o nível de deformação da armadura de aço obtida no dimensionamento (nes- te caso, $15,2 \%$ ) e $\varepsilon_{\text {sy }}$ é a deformação de escoamento da armadura de aço $(2,5 \%)$.

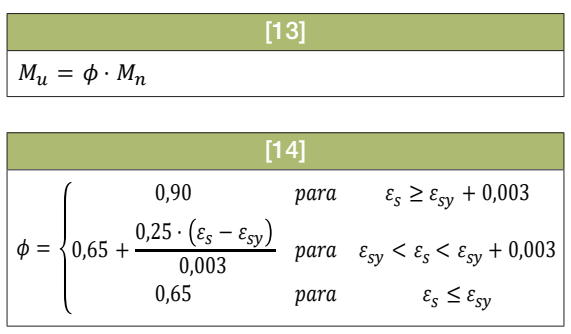

Considerando-se os resultados acima apresentados, $\phi$ é igual a 0,90. Assim:

$M_{u}=\phi \cdot M_{n}=0,90 \cdot 12,82 \mathrm{kN} \cdot m=11,54 \mathrm{kN} \cdot \mathrm{m}$

Tendo em vista a configuração de carregamento utilizada, este momento corresponde a uma carga concentrada, aplicada a meio vão, de 20,1 kN.

\section{I.3 ACl 440.IR (20I5)}

Conforme especificações da norma $\mathrm{ACl}$ 440.1R (2015), a tensão de tração máxima de projeto do compósito de FRP $\left(f_{f u}\right)$ depende da tensão fornecida pelo fabricante do FRP ou obtida em análises experimentais $\left(f_{\mathrm{fu}}^{\star}\right)$ e do coeficiente de exposição ambiental $\left(\mathrm{C}_{\mathrm{E}}\right)$, o qual é apresentado na Tabela 1.

A tensão de tração máxima no compósito, após a incidência do coeficiente de agressividade do meio, é dada pela Equação 15. Esta mesma análise é realizada para a deformação, cujo valor máximo de projeto do compósito de $\operatorname{FRP}\left(\varepsilon_{\mathrm{fu}}\right)$ também depende da deformação máxima do material, fornecida por ensaio ou por catálogo do fabricante $\left(\varepsilon_{\text {fu }}^{\star}\right)$, e da aplicação do coeficiente $C_{E}$ (Equação 16).

\begin{tabular}{ll|}
\hline & {$[15]$} \\
\hline$f_{f u}=f_{f u}^{*} \cdot C_{E}$ \\
\hline$\varepsilon_{f u}=\varepsilon_{f u}^{*} \cdot C_{E}$ \\
\hline
\end{tabular}

Neste caso, considerou-se que a viga dimensionada se encontra em um ambiente não exposto à água e intempéries e que será armada com barras de GFRP. Deste modo, o coeficiente $C_{E}$ utilizado é igual a 0,8 e as propriedades para dimensionamento são apresentadas nas Equações 17 e 18.

\begin{tabular}{c|}
\hline$[17]$ \\
\hline$f_{f u}=1047 \mathrm{MPa} \cdot 0,8=837,6 \mathrm{MPa}$ \\
\hline$[18]$ \\
\hline$\varepsilon_{f u}=21,8 \% 0 \cdot 0,8=17,4 \% 0$ \\
\hline
\end{tabular}

Para a definição do tipo de ruína, parâmetros como a taxa de armadura de FRP do elemento e a taxa de armadura balanceada (taxa de armadura para a qual ocorrem, simultaneamente, ruptura do FRP e esmagamento do concreto) são utilizados. O fator de redução $\beta_{1}$, apresentado pela ACl 440.1R (2015) e utilizado para o cálculo da taxa de armadura de balanceada de FRP pode ser calculado pela Equação 19, enquanto a taxa de armadura de FRP é calculada pela Equação 20 e a taxa de armadura balanceada pela Equação 21 , na qual $\varepsilon_{\text {cu }}$ é igual a $3 \%$. Como as

Tabela 1 - Fator ambiental de redução para variadas fibras e condições de exposição

\begin{tabular}{|c|c|c|}
\hline $\begin{array}{c}\text { Condição de } \\
\text { exposição }\end{array}$ & $\begin{array}{c}\text { Tipo de } \\
\text { fibra }\end{array}$ & $\begin{array}{c}\text { Fator } \\
\text { ambiental } \\
\text { de redução } \\
\left(\mathbf{C}_{E}\right)\end{array}$ \\
\hline $\begin{array}{c}\text { Concreto não } \\
\text { exposto } \\
\text { à água } \\
\text { e intempéries }\end{array}$ & Carbono & 1,0 \\
\hline $\begin{array}{c}\text { Concreto } \\
\text { exposto } \\
\text { à água }\end{array}$ & Aramida & 0,8 \\
\hline e intempéries & Aramidro & 0,9 \\
\hline Fonte: Adaptado de ACl 440.1R (2015) & 0,9 \\
\hline
\end{tabular}


barras de FRP não apresentam escoamento, a taxa de armadura balanceada é calculada com o uso da resistência à tração de projeto.

\begin{tabular}{|c|}
{$[19]$} \\
$\beta_{1}=0,85-\frac{0,05 \cdot\left(f_{c}^{\prime}-27,6\right)}{6,9} \geq 0,65$ \\
$\beta_{1}=0,85-\frac{0,05 \cdot(32,8-27,6)}{6,9}=0,812$ \\
\hline
\end{tabular}

$\quad[20]$
$\rho_{f}=\frac{A_{f}}{b \cdot d}$
$\rho_{f}=\frac{1,57 \mathrm{~cm}^{2}}{12 \mathrm{~cm} \cdot 17,5 \mathrm{~cm}}=0,0075$

\begin{tabular}{|c|}
\hline$[21]$ \\
$\rho_{f b}=0,85 \cdot \beta_{1} \cdot \frac{f_{c}^{\prime}}{f_{f u}} \cdot \frac{E_{f} \cdot \varepsilon_{c u}}{E_{f} \cdot \varepsilon_{c u}+f_{f u}}$ \\
$\rho_{f b}=0,85 \cdot 0,812 \cdot \frac{32,8 M P a}{837,6 M P a}$ \\
$\frac{48000 M P a \cdot 0,003}{48000 M P a \cdot 0,003+837,6 M P a}=0,0039$ \\
\hline
\end{tabular}
ada no esmagamento do concreto e o diagrama de tensões no concreto pode ser aproximado para a forma retangular apresentada no ACI 318 (2019), tal como apresentado na Figura 6. determinação da tensão de tração na barra é dada pela Equação 22.

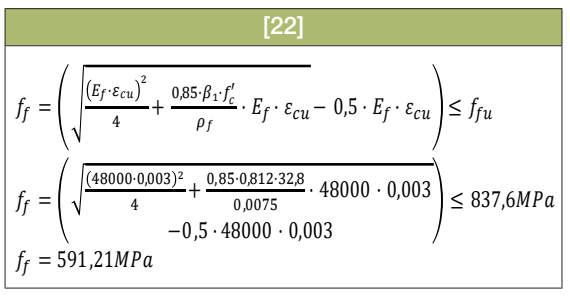

Quando $\rho_{f}>1,4 \cdot \rho_{\mathrm{fb}}$, a ruína é base-

Conforme o ACl 440.1R (2015), a

Sabe-se que o FRP é um material que apresenta comportamento elástico linear até a ruptura, e, desta forma, tendo-se a tensão de tração instalada na barra, pode ser utilizada a Lei de Hooke para determinação da deformação na armadura de FRP (Equação 23).

\begin{tabular}{|l|}
\hline \\
\hline$f_{f}=E_{f} \cdot \varepsilon_{f}$ \\
$591,21 M P a=48 G P a \cdot \varepsilon_{f} \rightarrow \varepsilon_{f}=12,32 \% 0$ \\
\hline
\end{tabular}

A profundidade da linha neutra (a) pode ser encontrada com o uso da Equação 24.

$a=\frac{A_{f} \cdot f_{f}}{0,85 \cdot f_{c}^{\prime} \cdot b}$
$a=\frac{1,57 \mathrm{~cm}^{2} \cdot 59,12 \mathrm{kN} / \mathrm{cm}^{2}}{0,85 \cdot 3,28 \mathrm{kN} / \mathrm{cm}^{2} \cdot 12 \mathrm{~cm}}=2,77 \mathrm{~cm}$

Por fim, multiplicando-se a força resultante na armadura e o braço de alavanca definido pelo binário de forças obtém-se a resistência nominal à flexão, como mostra a Equação 25.

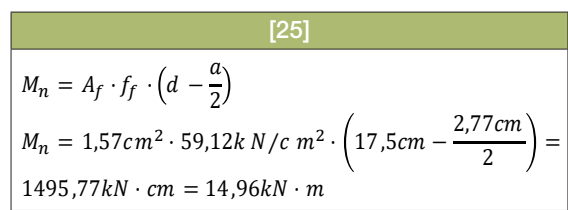

Tendo em vista a configuração de carregamento utilizada, este momento não minorado corresponde a uma carga concentrada, aplicada a meio vão, de $26,01 \mathrm{kN}$.

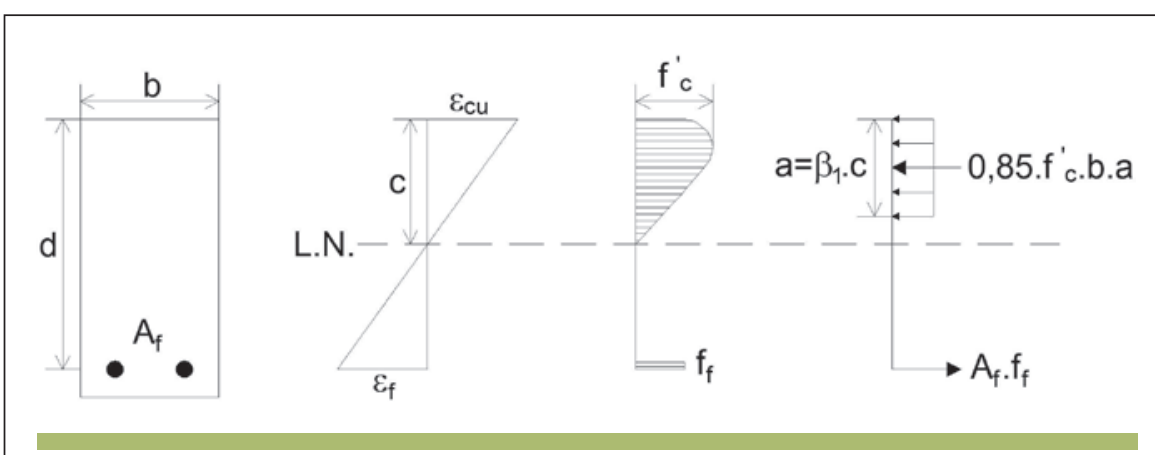

Figura 6

Diagrama aproximado retangular para esmagamento do concreto

Fonte: Adaptado de ACl 440.1R (2015)
O fator de redução da resistência, $\phi$, depende da razão entre a taxa de armadura de FRP e a taxa de armadura balanceada, como apresentado na Equação 26.

$\phi=\left\{\begin{array}{ccc|}0,55 & \text { se } & \rho_{f} \leq \rho_{f b} \\ 0,3+0,25 \cdot \frac{\rho_{f}}{\rho_{f b}} & \text { se } & \rho_{f b}<\rho_{f}<1,4 \cdot \rho_{f b} \\ 0,65 & \text { se } & \rho_{f} \geq 1,4 \cdot \rho_{f b} \\ \hline\end{array}\right.$

Assim, um fator de redução da resistência igual a 0,65 foi considerado. Deste modo, o momento calculado $\left(\mathrm{M}_{\mathrm{u}}\right)$ para a seção transversal é obtido seguindo o recomendado na Equação 27.

\begin{tabular}{|l|}
\hline \\
$\phi \cdot M_{n} \geq M_{u}$ \\
$0,65 \cdot 14,96 \geq M_{u}$ \\
$M_{u}=9,72 k N \cdot m$ \\
\hline
\end{tabular}

Tendo em vista a configuração de carregamento utilizada, este momento corresponde a uma carga concentrada, aplicada a meio vão, de 16,91 kN.

\subsection{Comparação entre 05 modelos de dimensionamento $\epsilon$ resultados experimentalmente obtidos}

Os resultados obtidos no programa de ensaios realizado por Mazzú (2020) são apresentados e discutidos de forma a avaliar a expectativa de capacidade resistente obtida via modelos de dimensionamento. Na Figura 7, apresenta-se a curva Força (F) versus deslocamento vertical na seção solicitada para as vigas com aço convencional e com barras de GFRP.

A Tabela 2 apresenta um resumo dos resultados obtidos no programa experimental para a força máxima e modo de ruptura observado. Nesta Tabela, $u_{F_{\text {máx }}}, \varepsilon_{\mathrm{Fmáx}}, \varepsilon_{\mathrm{F}_{\text {máx }}}$ e $\varepsilon_{F_{\text {mmáx }}}$ são o deslocamento na seção mais solicitada e deformações no concreto, na arma- 


\begin{tabular}{|c|c|c|c|c|c|c|}
\hline \multirow[b]{2}{*}{ Vigas } & \multicolumn{5}{|c|}{ Máxima força registrada } & \multirow[b]{2}{*}{ Modo de ruptura } \\
\hline & $\begin{array}{l}F_{\operatorname{máx}} \\
(\mathrm{kN})\end{array}$ & $\begin{array}{r}\mathrm{U}_{\text {Fmáx }} \\
(\mathrm{mm})\end{array}$ & 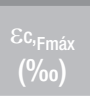 & $\begin{array}{c}\mathcal{E S}_{\text {, Fmáx }} \\
(\% 0)\end{array}$ & $\begin{array}{c}\mathcal{E f}_{\text {,Fmáx }} \\
(\% 0)\end{array}$ & \\
\hline V1-Aço & 25,35 & 49,21 & m.d. & m.d. & - & Escoamento do aço \\
\hline V2-Aço & 26,59 & 39,63 & m.d. & m.d. & - & Escoamento do aço \\
\hline V1-GFRP & 36,84 & 70,34 & m.d. & - & 14,88 & Esmagamento do concreto \\
\hline V-GFRP & 33,60 & 61,63 & m.d. & - & 14,10 & Esmagamento do concreto \\
\hline
\end{tabular}

dura de aço convencional e na armadura de GFRP, respectivamente, quando as vigas registraram o máximo carregamento $\left(F_{\text {máx }}\right)$. A Tabela 3 apresenta um comparativo entre os modelos teóricos, dimensionados com o uso das normas ABNT NBR 6118 (2014), ACl 318 (2019) e ACl 440.1R (2015) e os resultados experimentalmente obtidos.

As vigas com aço convencional ( $\mathrm{V} 1$ Aço e V2_Aço) apresentaram três estágios de comportamento bem definidos: (i) o primeiro representa o concreto não fissurado, cuja fissuração visível foi observada para uma força de aproximadamente 6 kN, (ii) o segundo corresponde ao concreto fissurado com o aço no regime elástico, o qual termina com força média de $23 \mathrm{kN}$, aproximadamente, e (iii) o terceiro equivale ao escoamento da armadura longitudinal de tração.

Com relação aos modelos teóricos de dimensionamento, os resultados obtidos com o uso das recomendações ABNT NBR 6118 (2014) e ACl 318 (2019) apresentam comportamentos bem semelhantes para determinação da capacidade resistente da seção transversal. Com relação às deformações, valores inferiores aos previstos com os modelos teóricos foram encontrados, entretanto, conduzindo a resultados seguros. Por fim, ambas as normas conseguiram prever com acerto os modos de ruptura verificados em laboratório.

As vigas com barras de fibras de vidro (V1_GFRP e V2_GFRP) apresentaram dois estágios de comportamento bem definidos: (i) o primeiro representa o concreto não fissurado, cuja fissuração visível foi observada para uma força de aproximadamente $5 \mathrm{kN}$, inferior a verificada para as vigas com armaduras de aço convencionais, e (ii) o segundo corresponde ao concreto fissurado com barras no regime linear, apresentando ruptura frágil ao atingir a força máxima.

Com relação aos modelos teóricos de dimensionamento, quando se remove o coeficiente de redução da resistência $(\phi)$, percebe-se que o valor de força nominal obtida com o ACl 440.1R (2015) se assemelha muito aos resultados obtidos para a viga de aço convencional. Entretanto, embora a viga experimentalmente avaliada atinja valores de força última

muito superiores ao obtidos com o uso de armaduras convencionais, o uso de $\phi$ faz com que os elementos armados com GFRP apresentem resistência de projeto de, no máximo, 16,9 kN (redução de $35 \%$ quando comparado aos calores característicos, sem $\phi$ ), indicando que os o uso da norma americana para o levantamento de capacidade resistente à flexão é conservadora e segura. Tais valores corroboram os obtidos por Mazzú (2020) na avaliação da capacidade resistente das vigas armadas com barras de GFRP e CFRP experimentalmente analisadas por Confrere et al. (2016).

Com relação às deformações, valores experimentais inferiores aos previstos com o modelo teórico foram encontrados

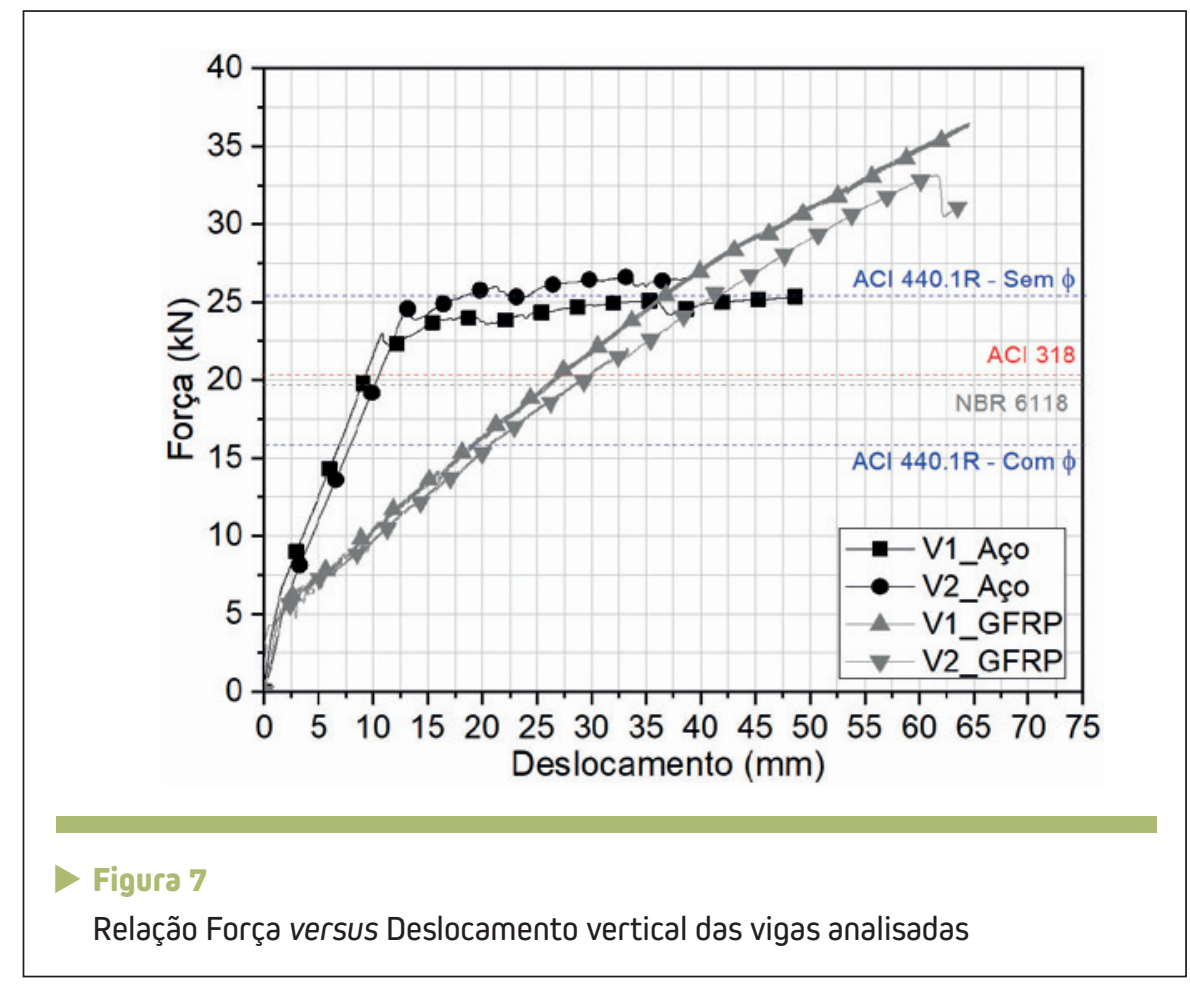




\begin{tabular}{|c|c|c|c|c|c|c|c|c|c|}
\hline \multirow{3}{*}{ Norma } & \multicolumn{6}{|c|}{ Modelos teóricos } & \multicolumn{3}{|c|}{ Programa experimental } \\
\hline & \multicolumn{5}{|c|}{ Dimensionamento ELU } & \multirow[b]{2}{*}{ Modo de ruptura } & \multirow[b]{2}{*}{$\begin{array}{c}\varepsilon c \\
(\% 0)\end{array}$} & \multirow[b]{2}{*}{$\begin{array}{c}\text { Es } \\
(\% 0)\end{array}$} & \multirow[b]{2}{*}{$\begin{array}{c}\text { Ef } \\
(\% 0)\end{array}$} \\
\hline & $\begin{array}{c}\mathrm{M} \\
(\mathrm{kN} \cdot \mathrm{m})\end{array}$ & $\begin{array}{c}F \\
(\mathrm{kN})\end{array}$ & $\begin{array}{c}\varepsilon_{0} \\
(\% 0)\end{array}$ & $\begin{array}{c}\varepsilon s \\
(\% \circ)\end{array}$ & $\begin{array}{c}\text { \&f } \\
(\% 0)\end{array}$ & & & & \\
\hline NBR 6118 & 10,97 & 19,10 & $-2,6$ & 10,0 & - & Escoamento da armadura & $-0,7$ & 1,8 & - \\
\hline ACl 318 & 11,54 & 20,10 & $-3,0$ & 15,2 & - & Escoamento da armadura & $-1,3$ & 2,1 & - \\
\hline \multirow{2}{*}{$\mathrm{ACl} 440.1 \mathrm{R}$} & 9,72 & $16,9^{2}$ & $-3,0$ & - & 12,3 & \multirow{2}{*}{ Esmagamento do concreto } & $-1,4$ & - & 6,7 \\
\hline & 14,96 & $26,0^{1}$ & $-3,0$ & - & 12,3 & & $-2,9$ & - & m.d. \\
\hline
\end{tabular}

${ }^{1}$ Sem redução e ${ }^{2}$ Com redução da resistência com o uso de $\phi$; m.d. - extensômetros utilizados na campanha experimental para obtenção das deformações foram mecanicamente danificados

para a capacidade resistente de projeto (16,9 kN). Por fim, com relação ao modo de ruptura, o modelo apresentado pela $\mathrm{ACl}$ 440.1R (2015) conseguiu prever o modo de ruptura das vigas com barras de GFRP analisada neste trabalho, a qual foi baseada no esmagamento do concreto comprimido.

\section{CONCLUSÕES}

A utilização de barras de aço como armadura ativa e passiva em estruturas de concreto armado é muito comum na indústria da construção civil no Brasil. Entretanto, problemas relacionados principalmente à corrosão das armaduras de aço pela localização de estruturas de concreto em zonas de alta agressividade ambiental tem levado ao desenvolvimento de novas técnicas de proteção ou a novos materiais, tais como o uso de barras de FRP como armadura. Neste sentido, este trabalho apresentou o dimensionamento à flexão de vigas bi apoiadas de concreto armadas com barras de aço convencionais e de GFRP, cujos resultados foram comparados aos obtidos em ensaios.

A partir dos resultados obtidos neste trabalho, conclui-se que:

> O dimensionamento baseado nas normas ABNT NBR 6118 (2014) e ACl 318 (2019) mostram-se analiticamente eficazes para determinar, com segurança, a capacidade de carga de vigas de concreto armado armadas com aço convencional e seu modo de ruptura;

> Os modelos apresentados permitem dimensionamento seguro dos elementos de concreto armados com barras de GFRP, ainda que de forma conservadora desde que considerado o coeficiente de redução $\phi$;

A norma ACl 440.1R (2015) também conseguiu prever corretamen- te o modo de ruptura das vigas armadas com barras de GFRP; e

> Com relação às deformações, valores inferiores aos previstos com os modelos teóricos foram encontrados, entretanto, conduzindo a resultados seguros.

Note-se que a utilização de armadura de FRP em estruturas de concreto armado ainda não é comum no Brasil, ainda não existem muitas pesquisas nesta linha e ainda não existe normativa brasileira que balize a utilização deste tipo de material no país. Desta forma, os resultados aqui apresentados estão limitados à série de vigas ensaiadas no presente trabalho, porém, é necessária a realização de um programa experimental mais abrangente visando a compreensão do comportamento de elementos armados com barras de FRP e das potencialidades do uso deste material, principalmente quando aplicado em ambientes agressivos.

\section{DREFERÊNCIAS BIBLIOGRÁFICAS}

[1] AMERICAN CONCRETE INSTITUTE. ACI 318. Building code requirements for reinforced concrete. Detroit, USA. 2019

[2] AMERICAN CONCRETE INSTITUTE. ACI COMMITTEE 440.1R. Guide for the Design and Construction of Structural Concrete Reinforced with Fiber-Reinforced Polymer (FRP) Bars. 2015.

[3] AMERICAN CONCRETE INSTITUTE. ACI COMMITTEE 440.9R. Guide to Accelerated Conditioning Protocols for Durability Assessment of Internal and External Fiber-Reinforced Polymer (FRP) Reinforcement. 2015.

[4] ASSOCIAÇÃO BRASILEIRA DE NORMAS TÉCNICAS. NBR 6118. Projeto de estruturas de concreto: Rio de Janeiro, 2014.

[5] CONFRERE, A.; MICHEL, L.; FERRIER, E.; CHANVILLARD, G. (2016). Experimental behaviour and deflection of low-strength concrete beams reinforced with FRP bars. Structural Concrete, v. 17, n. 5, p. $858-874$.

[6] DALFRÉ, G.M.; FERREIRA, F.G.S.; MAZZÚ, A.D.E. Dimensionamento de vigas de concreto armadas à flexão com barras não metálicas. Revista CONCRET0 \& Construções, V. XLVIII, 98, pp. 72-78.

[7] ISSA, M. S.; METWALLY, I. M.; ELZEINY, S. M. (2011). Influence of fibers on flexural behavior and ductility of concrete beams reinforced with GFRP rebars. Engineering Structures, v. 33, pp. 1754-1763.

[8] LIMA, R. S.; PRADO, F. S. Análise numérica de vigas de concreto armadas com barras de fibra de vidro (GFRP) e aço. In: SEMINÁRIO MAUÁ DE INICIAÇÃO CIENTÍFICA, 6, 2014, São Caetano do Sul. Anais... São Caetano do Sul, 2014.

[9] MAZZÚ, A. D. E. (2020). Estudo sobre a substituição de armadura metálica por barras de GFRP em vigas de concreto armadas à flexão quando submetidas ao ataque acelerado de íons cloreto. 2020. 190p. Dissertação (Mestrado em Engenharia Civil) - Universidade Federal de São Carlos, São Carlos, 2020. 